\title{
Chaotic diffusion caused by close encounters with several massive asteroids
}

\section{The regions of (10) Hygiea, (2) Pallas, and (31) Euphrosyne ${ }^{\star}$}

\author{
V. Carruba ${ }^{1}$, M. Huaman ${ }^{1}$, R. C. Domingos ${ }^{2}$, and F. Roig ${ }^{3}$ \\ 1 UNESP, Univ. Estadual Paulista, Grupo de dinâmica Orbital e Planetologia, Guaratinguetá, SP, 12516-410, Brazil \\ e-mail: vcarruba@feg.unesp.br \\ 2 INPE, Instituto Nacional de Pesquisas Espaciais, São José dos Campos, SP, 12227-010, Brazil \\ 3 ON, Observatório Nacional, Rio de Janeiro, RJ, 20921-400, Brazil
}

Received 26 September 2012 / Accepted 12 December 2012

\begin{abstract}
Context. Close encounters with (1) Ceres and (4) Vesta, the two most massive bodies in the main belt, are known to be a mechanism of dynamical mobility able to significantly alter proper elements of minor bodies, and they are the main source of dynamical mobility for medium-sized and large asteroids ( $D>20 \mathrm{~km}$, approximately). Recently, it has been shown that drift rates caused by close encounters with massive asteroids may change significantly on timescales of $30 \mathrm{Myr}$ when different models (i.e., different numbers of massive asteroids) are considered.

Aims. So far, not much attention has been given to the case of diffusion caused by the other most massive bodies in the main belt: (2) Pallas, (10) Hygiea, and (31) Euphrosyne, the third, fourth, and one of the most massive highly inclined asteroids in the main belt, respectively. Since (2) Pallas is a highly inclined object, relative velocities at encounter with other asteroids tend to be high and changes in proper elements are therefore relatively small. It was thus believed that the scattering effect caused by highly inclined objects in general should be small. Can diffusion by close encounters with these asteroids be a significant mechanism of long-term dynamical mobility?

Methods. By performing simulations with symplectic integrators, we studied the problem of scattering caused by close encounters with (2) Pallas, (10) Hygiea, and (31) Euphrosyne when only the massive asteroids (and the eight planets) are considered, and the other massive main belt asteroids and non-gravitational forces are also accounted for.

Results. By finding relatively small values of drift rates for (2) Pallas, we confirm that orbital scattering by this highly inclined object is indeed a minor effect. Unexpectedly, however, we obtained values of drift rates for changes in proper semi-major axis $a$ caused by (10) Hygiea and (31) Euphrosyne larger than what was previously found for scattering by (4) Vesta. These high rates may have repercussions on the orbital evolution and age estimate of their respective families.
\end{abstract}

Key words. minor planets, asteroids: general - minor planets, asteroids: individual: (10) Hygiea - celestial mechanics minor planets, asteroids: individual: (31) Euphrosyne - minor planets, asteroids: individual: (2) Pallas

\section{Introduction}

Orbital mobility caused by close encounters with massive asteroids has been studied in the past and could be a viable mechanism to produce the current orbital location of some of the V-type asteroids presently outside the Vesta family. It is well known, however, that the proper frequencies of precession of pericenter $g$ and longitude of the node $s$ of terrestrial planets change when one or more of the other planets is not considered in the integration scheme. For instance, the $g_{4}$ and $s_{4}$ frequencies of Mars are different when the full solar system is considered or when only Mars and the Jovian planets are accounted for. Carruba et al. (2012) showed that (4) Vesta proper frequencies are dependent on the number of other massive asteroids included in the integration scheme and that, as a result, the whole statistics of encounters with (4) Vesta is also affected.

Delisle \& Laskar (2012) showed that dynamical mobility caused by close encounters with massive asteroids is dominated

* Appendix $\mathrm{A}$ is available in electronic form at http: //www . aanda.org by encounters with (4) Vesta and (1) Ceres, with the other massive asteroid playing a lesser role. Previous studies on this subject (Carruba et al. 2003, 2007) also focused on the dynamical mobility caused by the two largest bodies. Can the third, fourth, or other most massive bodies have also played a role in the dynamical mobility of minor bodies, at least in their orbital proximities? And if that is possible, how much is the drift rate in semimajor axis affected by the choice of the model used to simulate the long-term effect of close encounters with massive asteroids, as observed in the region of (4) Vesta (Carruba et al. 2012)?

In this work we will focus on the dynamical mobility caused by close encounters with the third ((2) Pallas) and the fourth ((10) Hygiea) asteroid in the main belt, with attention on the scattering effect on members of their respective families. Since one of the goals of this investigation is to study the long-term effect of close encounters with highly inclined massive asteroids, we also considered the case of (31) Euphrosyne. Previous studies (Baer et al. 2011) have indicated that this body was the fifth-largest in the main belt. New estimates (Carry 2012) have considerably reduced its mass, but it remains one of 
the most massive bodies that are part of a significantly large asteroid family (Machuca \& Carruba 2011). Since the population of large asteroids is somewhat limited in the orbital proximity of (2) Pallas, we studied the long-term orbital diffusion caused by close encounters with this body also to better assess how relevant this effect is for highly inclined asteroids. In particular, if we consider the frequency distribution function of relative velocities at close encounters for highly inclined asteroids, does there exist a significant statistical tail for which relative velocities are small enough that large changes in proper semi-major axis $a$ can be observed?

This work is structured as follows: in Sect. 2 we study the orbital neighborhood of (2) Pallas, (10) Hygiea, and (31) Euphrosyne by obtaining dynamical maps. Section 3 is dedicated to estimating the changes in the proper frequencies of these massive asteroids observed when different integration schemes are considered. In Sect. 4 we obtain the statistics of changes in proper semi-major axis caused by close encounters only when different models of the main belt are considered. In Sect. 5 we discuss the statistical completeness of the frequency distribution functions that we obtained in Sect. 4. In Sect. 6 we also include in our models non-gravitational forces such as the Yarkovsky and YORP effects. Finally, in Sect. 7 we present our conclusions.

\section{Dynamical maps}

We start our analysis by studying the orbital regions where the massive asteroids of interest are currently located in the main belt. With a mass of $8.63 \times 10^{19} \mathrm{~kg}$, (10) Hygiea is the fourth most massive body in the main belt. According to Milani \& Knežević (1994), (10) Hygiea and its family reside in a fairly stable area of the outer main belt, between the 9J:-4A and the 2J:-1A mean-motion resonances in semi-major axis. The most notable secular resonance in the area is the $z_{1}=g-g_{6}+s-s_{6}$, that, computed for the values of proper $a, e$, and $i$ of (10) Hygiea, crosses a region just above the typical inclination values of Hygiea family members.

To confirm this scenario, we obtained a dynamical map of synthetic proper elements, computed with the approach of Knežević \& Milani (2003), Carruba (2010), for the region of (10) Hygiea. We took 6464 particles between 2.950 and $3.265 \mathrm{AU}$ in semi-major axis, $0^{\circ}$ and $10^{\circ}$ and in $i$, with a stepsize of $0.005 \mathrm{AU}$ in $a$ and $0.1^{\circ}$ in $i$. All the other elements of the test particles were those of (10) Hygiea at the osculating date of J2000. Our results are shown in Fig. 1, where the test particles appear as black dots. Mean-motion resonances will appear in this map as vertical strips with lower densities of test particles, while secular resonances appear as elongated diagonal alignments of bodies (the names of secular resonances in the region are shown in red). Regular regions show a uniform population of test particles. The position of (10) Hygiea itself is shown as a magenta full dot. We also show in the figure the orbital position of all real asteroids with absolute magnitudes $H<13.5$ in the same $a-i$ range of our test particles as blue asterisks ${ }^{1}$. As in Carruba et al. (2012), we choose only the largest bodies because asteroids with diameters larger than $\simeq 20 \mathrm{~km}$ are the ones for which close encounters are the dominant mechanism of dynamical mobility on

\footnotetext{
The dynamical map was computed using the orbital elements of (10) Hygiea at J2000. The location of secular resonances may vary when computed for the orbits of other asteroids. See also Carruba \& Morbidelli (2011).
}

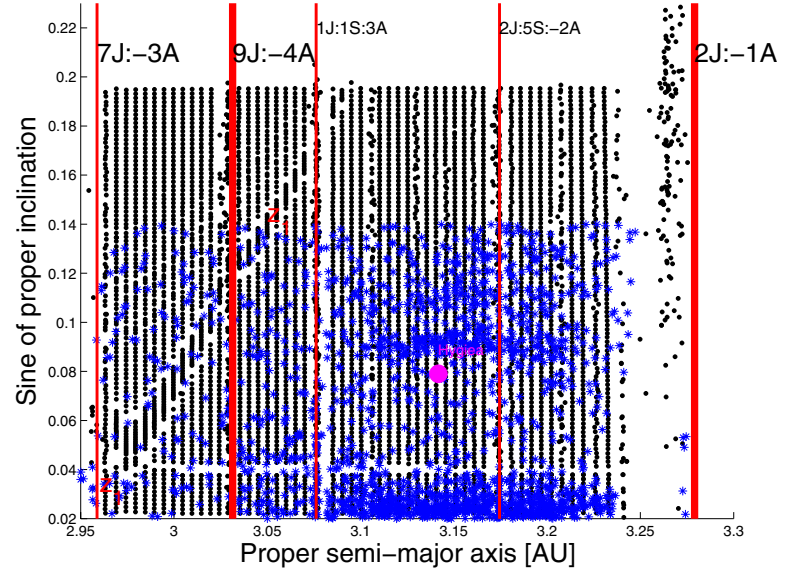

Fig. 1. Dynamical map of 6464 test particles in the region of the Hygiea family (black dots). We note the presence of the $z_{1}$ secular resonance, which appears as an elongated diagonal strip of aligned test particles, near the Koronis family. Real asteroids in the region with $H<13.5$ are shown as blue asterisks, the orbital position of (10) Hygiea itself appears as a magenta full dot.

timescales of $250 \mathrm{Myr}$, according to Delisle \& Laskar (2011; see also a discussion in Carruba et al. 2003). Using the relationship

$D(\mathrm{~km})=1329 \frac{10^{(-H / 5)}}{\sqrt{p_{V}}}$,

where $p_{V}$ is the geometric albedo, equal approximately to 0.05 for typical C-type objects and 0.1 for typical S-type objects (Bowell et al. 1989), we find that $H=13.5$ corresponds to diameters of approximately $11.86 \mathrm{~km}$ for C-type objects and $8.39 \mathrm{~km}$ for S-type objects, respectively. These diameters are still in the regime where orbital mobility caused by close encounters is not negligible when compared with the mobility caused by the Yarkovsky effect.

To avoid including Koronis family members we only selected $\sin (i)<0.14$ asteroids for the Hygiea region. The Hygiea family lies in a fairly regular region, at about 0.1 in $\sin (i)$. We then considered the region of (2) Pallas. We took 5000 particles between 2.7 and $2.85 \mathrm{AU}$ in semi-major axis, $25^{\circ}$ and $48^{\circ}$ in $i$, with a step-size of $0.0025 \mathrm{AU}$ in $a$ and $0.2^{\circ}$ in $i$. All the other elements of the test particles were those of (2) Pallas at J2000. Our results are shown in Fig. 2, where the test particles appear as black dots. The position of (2) Pallas itself is shown as a magenta full dot. We also show in the figure the orbital position of all real asteroids with absolute magnitudes $H<13.5$ in the same $a-i$ range of our test particles.

Finally, we investigate the case of (31) Euphrosyne. We took 4500 particles between 3.02 and 3.32 AU in semi-major axis, $20^{\circ}$ and $35^{\circ}$ in $i$, with a step-size of 0.005 AU in $a$ and $0.2^{\circ}$ in $i$. All the other elements of the test particles were those of (31) Euphrosyne at J2000. Our results are shown in Fig. 3, where the test particles appear as black dots. The position of (31) Euphrosyne itself is shown as a magenta full dot. All real asteroids with absolute magnitudes $H<13.5$ in the same $a-i$ range of our test particles are also shown in the figure.

Euphrosyne lies above the more highly populated region of the Alauda family and is crossed by a rich web of linear and non-linear secular resonances (see Machuca \& Carruba 2011 , for a more in-depth discussion of the local dynamics and family determination). Now that we have studied the problem of local dynamics, we may start quantifying the indirect effect that the presence of other asteroids may have on the drift 


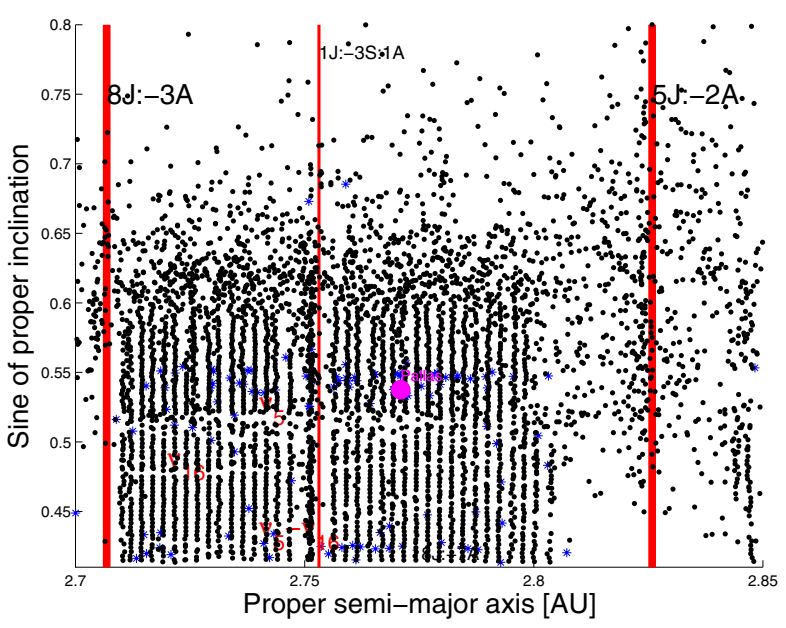

Fig. 2. Dynamical map of 5000 test particles in the region of the Pallas family (black dots). The orbital location of (2) Pallas is shown as a magenta dot, the other symbols are the same as in Fig. 1.

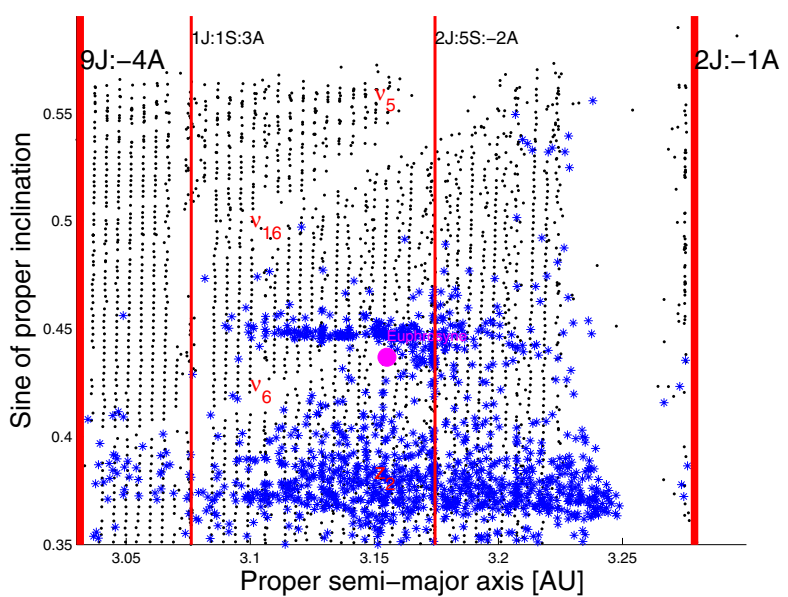

Fig. 3. Dynamical map of 4500 test particles in the region of the Euphrosyne family (black dots).

rates caused by close encounters with (2) Pallas, (10) Hygiea, and (31) Euphrosyne. This is the subject of the next section.

\section{The indirect effect of other massive asteroids: choosing the right model}

To investigate for possible indirect effects caused by the presence of other massive asteroids in the model, we obtained the values of the proper frequencies of pericenter and longitude of the node precession $g$ and $s$ for (2) Pallas, (10) Hygiea, and (31) Euphrosyne for simulations involving progressively all 39 main belt asteroids more massive than $8.5 \times 10^{18} \mathrm{~kg}$ in the Carry (2012) paper $^{2}$. The proper frequencies were computed with the frequency modified Fourier transform method of Laskar (1988, 1990, 1993), using an algorithm also described in Šidlichovský \& Nesvorný (1997). A list of the first 39 most massive asteroids is given in Table A.1. For the mass of (4) Vesta,

\footnotetext{
2 The first simulation for obtaining Pallas frequencies had the eight planets plus (2) Pallas (S1 model), the second also included (1) Ceres (S2 model), the third included all of the above plus (4) Vesta (S3 model), and so on.
}

we used the value obtained from the DAWN mission (Russel et al. 2012). Following the approach of Carruba et al. (2012), we integrated our asteroids as massive bodies over $30 \mathrm{Myr}$ with SWIFT-SKEEL, the symplectic integrator using the Wisdom and Holman mapping (Wisdom \& Holman 1991; Levison \& Duncan 2000). Results for the $g$ frequency (for simplicity we do not show the $s$ data) are shown in Fig. 4, panels A, C, and E. For each of the 39 simulations with massive asteroids, we computed the values of the $g$ and $s$ frequencies over four time intervals of $8.192 \mathrm{Myr}$ using the approach of Knežević \& Milani (2003). We took the mean of the four values as an estimate of the proper frequencies and the standard deviation as an estimate of the error. Vertical black lines display the error associated with each frequency value, the blue horizontal line shows the mean value of $g$ for all the 39 simulations, and the horizontal red lines display the values of frequencies between the mean value minus and plus the standard deviation of the data (we refer to this interval as the "confidence level"). Panels B, D, and F of Fig. 4 display the number of close encounters among massive asteroids as a function of the number of massive asteroids included in the model.

For the case of (10) Hygiea, the number of close encounters experienced by this asteroid during the simulation was somewhat limited when compared with results observed for (4) Vesta (Carruba et al. 2012). The models for which this number was the highest correspond to values of $g$ frequencies beyond a $3 \sigma$ level, but not to the highest (or lowest) peaks (models with 12 and 14 asteroids, for instance). Mechanisms other than close encounters with massive asteroids, possibly such as secular perturbation of the precession frequency of (10) Hygiea by other massive asteroids, should be at play to explain the different values of secular frequencies in these cases. The first model for which the frequency was close to the mean value occurred for six asteroids, while the first peak in the $g$ frequency for models with more than four asteroids was observed for nine asteroids.

In the (2) Pallas case, while the number of close encounters was higher than in the (10) Hygiea one, we still do not see a clear correspondence between the models with the largest number of close encounters and the peaks in the $g$ distribution. The largest peaks (models S16 and S28) are not associated with close-encounter events. The first model with more than four asteroids for which the frequency was close to the mean value was the S6, while the first peak in the $g$ frequency was observed in the S9.

Finally, (31) Euphrosyne, more than (10) Hygiea and (2) Pallas, experienced only a very limited number of close encounters with massive asteroids. The first model for which the frequency was close to the mean value occurred for seven asteroids, while the first peak in the $g$ frequency for models with more than five asteroids (the four most massive plus (31) Euphrosyne) were observed for six asteroids.

Table 1 summarizes our results for the three massive asteroids that we investigated. The second column shows the model for which the asteroid $g$ frequency was closer to the mean value, and the third column the model for which the first peak beyond a $3 \sigma$ level was observed. We will use these models in Sect. 4 to investigate the long-term behavior of chaotic diffusion caused by close encounters with these massive asteroids.

\section{Chaotic diffusion caused by close encounters}

To obtain the statistics of changes in proper $a$ caused by close encounters with the three considered massive asteroids, we used 
(10) Hygiea

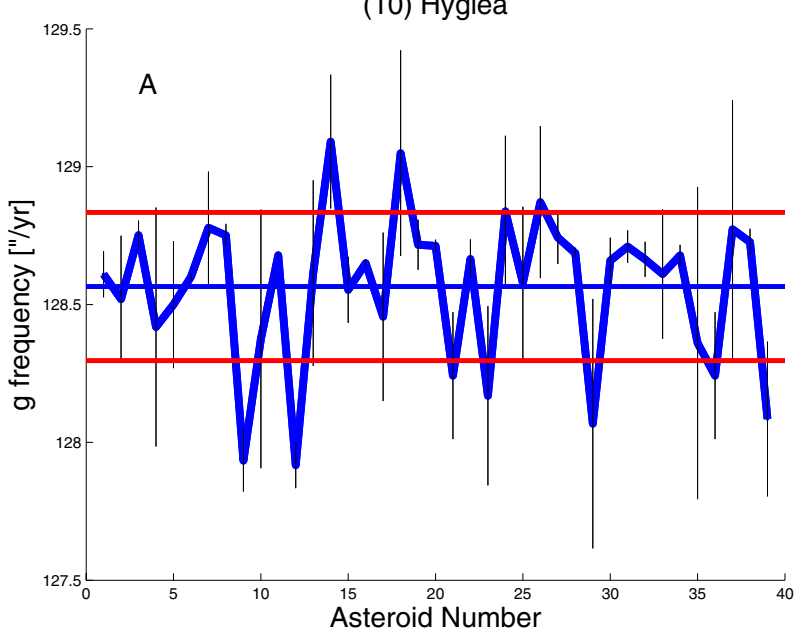

(2) Pallas

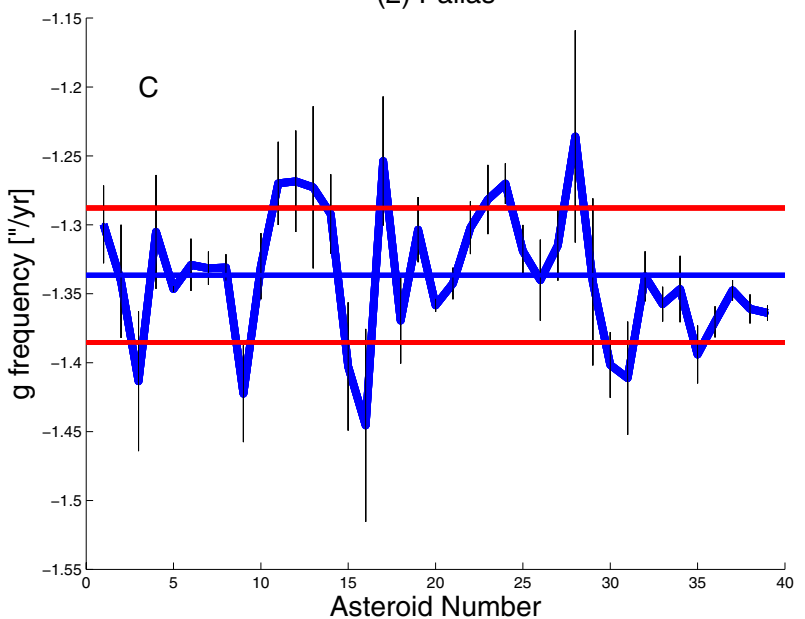

(31) Euphrosyne

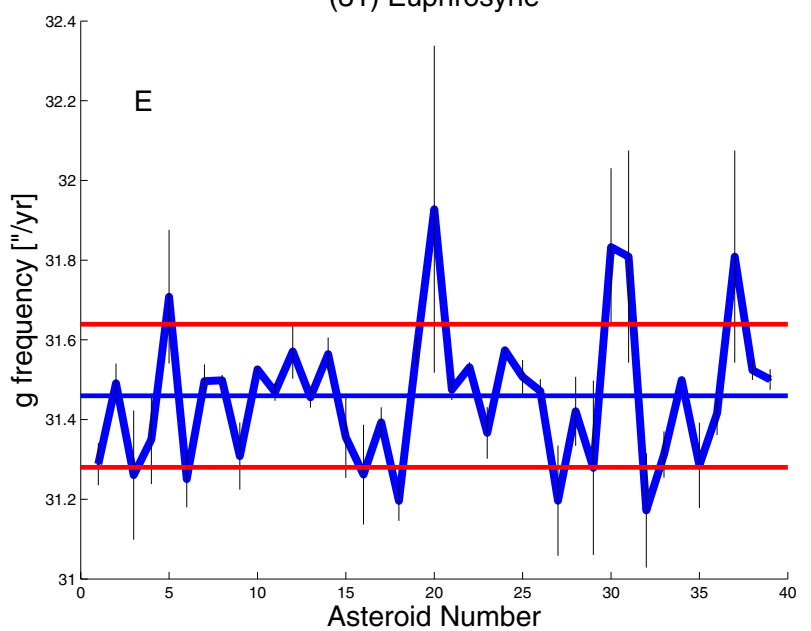

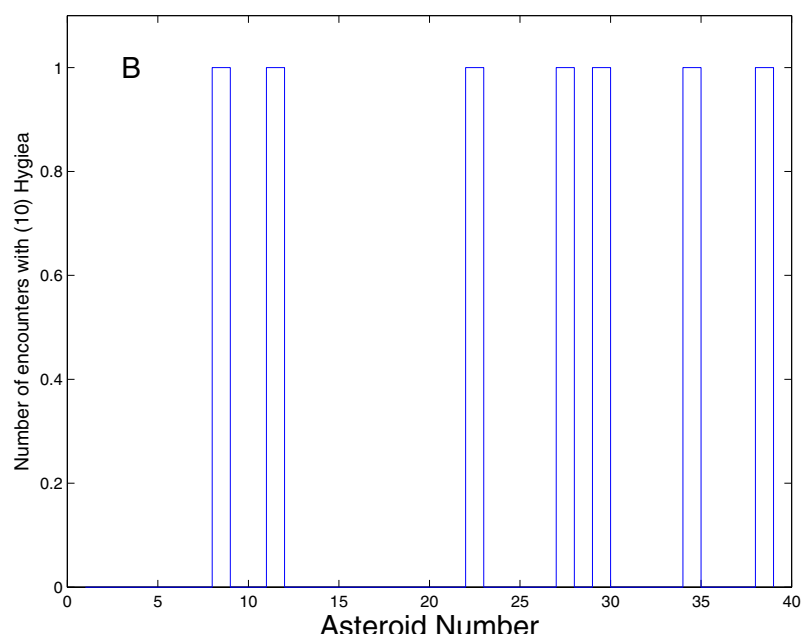
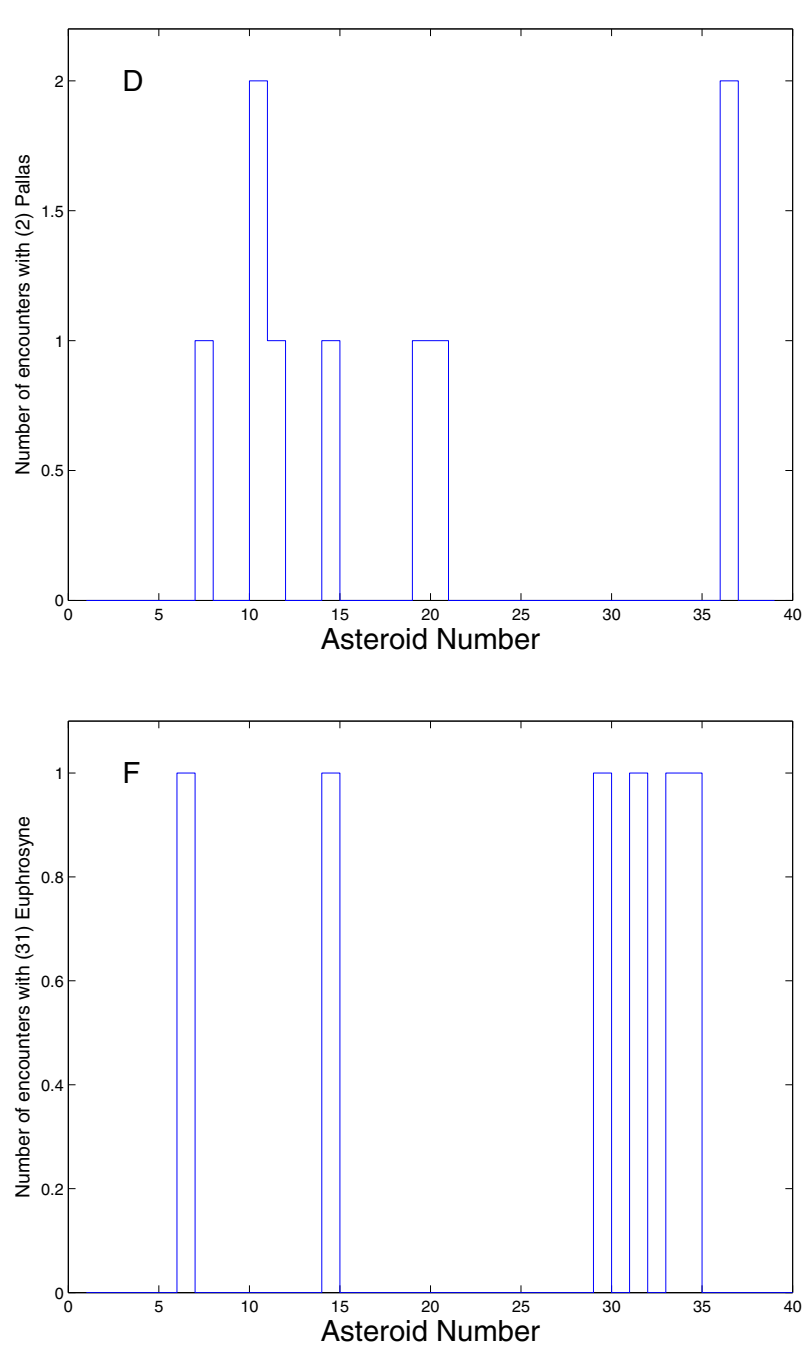

Fig. 4. Panels A), C), E) dependence of the precession frequency of the Hygiea, Pallas, and Euphrosyne pericenter $g$ on the number of other massive asteroids included in the simulation. Asteroids are given in the order of Table A.1. Panels B), D), F) number of close encounters among massive asteroids as a function of the number of asteroids in the model.

the SWIFT-SKEEL code of the SyMBA package of Levison \& Duncan (2000). We integrated our asteroids over $30 \mathrm{Myr}$ and used the S0 simulation to estimate the noise in the change in proper $a$ caused by secular effects with the method described in Delisle \& Laskar (2012). Since the Bienaymé formula states that the variance of the sum (or the difference) of uncorrelated random variables is the sum of their variances, the variance in jump sizes obtained from a numerical simulation with massive 
Table 1. Integration schemes for which the asteroid $g$ frequency was closer to the mean value (second column) and for which the first peak beyond a $3 \sigma$ level was observed.

\begin{tabular}{lcc}
\hline \hline Asteroid & First mean value & First peak in $g$ \\
\hline (10) Hygiea & S6 & S9 \\
(2) Pallas & S6 & S9 \\
(31) Euphrosyne & S7 & S6 \\
\hline
\end{tabular}

asteroids will be the sum of the variance of the real diffusion and the variance of the noise. Therefore, the real diffusion resulting from close encounters will be given by

$\bar{\sigma}_{a}^{2}[1]=\sigma_{a}^{2}[1]-\sigma_{\text {noise }}^{2}[1]$

We used the results of the SO simulation to estimate the value of the variance caused by the noise. As in Carruba et al. (2012), we found a value of the standard deviation, the square root of the variance, equal to $\sigma_{\text {noise }} \simeq 2 \times 10^{-4} \mathrm{AU}$. Since the stronger changes in proper $a$ (identified as $\Delta a$ hereafter) are those that i) are more significant for the dynamical mobility caused by close encounters with massive asteroids (Carruba et al. 2003); and ii) are those that are less likely to be caused by secular effects, we concentrated our attention on the values of $\Delta a>3 \sigma_{\text {noise }}=$ $6 \times 10^{-4} \mathrm{AU}$.

We also computed the skewness, kurtosis higher moments of the distributions of changes in proper $a$, and the The Hurst exponent $T$ of the changes in $\bar{\sigma}_{a}[1]$. The skewness gives a measure of the asymmetry of the probability distribution of a real-value random variable, while the kurtosis is any measure of the "peakedness" of the probability distribution of a real-value random variable (see Carruba et al. 2012, for a more in-depth description of these parameters). We assumed that the Hurst exponent could be fitted by a power law of the time of the form

$\bar{\sigma}_{a}[1](t)=C t^{\mathrm{T}}$,

where $C$ is a constant. The Hurst exponent gives a measure of how much the diffusion process differs from a random walk. It is equal to 0.5 for random walk-like processes, and is between 0.5 and 1.0 for correlated and persistent processes. Previous values of $T$ for diffusion caused by close encounters with (1) Ceres for members of the Adeona and Gefion families were of the order of 0.58-0.72 (Carruba et al. 2003). Here we compute this exponent using Eq. (3) to best fit $T$ using three values of $\bar{\sigma}_{a}[1](t)$ computed at each $10 \mathrm{Myr}$. For each of our simulations, we therefore computed the following parameters: the total number of encounters, the number of encounters for which $\Delta a$ was larger than $3 \sigma_{\text {noise }}$, the reduced standard deviation $\bar{\sigma}_{a}[1]$ without the "noise" of the simulation, obtained from Eq. (2), the skewness, kurtosis, and Hurst exponent. We start our analysis by investigating the Hygiea region.

\section{1. (10) Hygiea}

For the region of (10) Hygiea, we simulated the 2914 real $H<$ 13.5 asteroids under the influence of all planets plus (10) Hygiea with a time-step of two days (see Carruba et al. 2012, for a discussion on the choice of this parameter), and we monitored during the simulation if the distance from this asteroid was less than $0.001 \mathrm{AU}$ (see discussion in Carruba et al. 2012, for the choice of this parameter). We used five integration schemes: S0, S1, S4, and the ones whose proper frequencies $g$ and $s$ differed most from the mean value (S6 and S9, respectively). As in Carruba et al. (2012), we found that the same asteroids did not experience close encounters in different integration schemes (for instance, asteroids that experienced large changes in $a$ in one integration scheme may not have experienced close encounters at all in another) and that there were fluctuations in the statistics of $\Delta a$ as well.

Table 2 summarizes our results. Histograms of frequencies of changes of proper $a\left(N_{i} / N_{\text {tot }}\right.$, where $N_{i}$ is the number of encounters in the $i$ th bin and $N_{\text {tot }}$ is the total number of encounters) for the four integration schemes used are also shown in Fig. 5, panel A. Unexpectedly, we found large values of $\bar{\sigma}_{a}[1]$ caused by encounters with (10) Hygiea: the mean value of $90.8 \times 10^{-5} \mathrm{AU}$ is even larger than the corresponding mean value of $\bar{\sigma}_{a}[1]=(78.6 \pm 15.6) \times 10^{-5}$ AU caused by encounters with (1) Ceres, the main perturber in the main belt, in the (4) Vesta region as found in Carruba et al. (2012). We also found that $12.4 \%$ of the encounters satisfied our criterion for relevance of causing a change in $\Delta a$ larger than $3 \sigma=6 \times 10^{-4} \mathrm{AU}$. This is by far the largest percent fraction found in our simulations so far. The unexpected high rate of drift in proper $a$ caused by encounters with (10) Hygiea, unknown in previous works in the literature, may have played a yet to be investigated significant role in the evolution of the Hygiea family.

\section{2. (2) Pallas}

For the region of (2) Pallas, we simulated the 300 real $H<$ 13.5 asteroids under the influence of all planets plus (2) Pallas, using the same integration schemes as discussed in Sect. 4.1. The limited number of known objects in the Pallas region is caused by the fact that this is a highly inclined region well above the $v_{6}$ and $v_{5}$ secular resonances (see Carruba \& Machuca 2011, for a more in-depth discussion on the highly inclined asteroid population in stable and unstable areas). We used the standard S1 and S4 integration schemes, plus the S6 and S9 schemes associated with the first mean value and the first peak in the $g$ frequency of (2) Pallas discussed in Sect. 3. Results for the $\Delta a$ statistics caused by close encounters with (2) Pallas are summarized in Table 2. A histogram of frequency of $\Delta a$ values is shown in Fig. 5, panel B.

Contrary to what was found for (10) Hygiea, we found the rather low value of mean $\bar{\sigma}_{a}[1]\left(20.8 \times 10^{-5} \mathrm{AU}\right)$, just a factor two higher than what was found for encounters with (2) Pallas in the Vesta region in Carruba et al. (2012). Only a very limited number of close encounters satisfied our criterion for relevance of causing a change in $\Delta a$ larger than $3 \sigma$ (at most, just three for the S9 simulation). The very low value of drift in proper $a$ caused by encounters with (2) Pallas seems to confirm the hypothesis of Bottke et al. (1994) that encounters with this highly inclined asteroid occur mostly at high relative speed and distances and therefore cause limited changes in proper elements. To further investigate this hypothesis, we consider the case of (31) Euphrosyne in the next sub-section.

\section{3. (31) Euphrosyne}

For the region of (31) Euphrosyne, we simulated the 2138 $H<13.5$ asteroid under the influence of all planets plus (31) Euphrosyne, using the integration schemes listed in Table 2. Results are also given in Table 2, with a histogram of frequency of $\Delta a$ values shown in Fig. 5, panel C. 
Table 2. Numbers of encounters, numbers of encounters with $|\mathrm{d} a|>3 \sigma_{\text {noise }}$, and moments (in $\mathrm{AU}$ ) of the distribution of changes in proper $a$ caused by (10) Hygiea, (2) Pallas, and (31) Euphrosyne in the S1, S4, first mean value, and first peak integrations.

\begin{tabular}{lccccccc}
\hline \hline Asteroid & Simulation & Number of encounters & $N\left(|\mathrm{~d} a|>3 \sigma_{\text {noise }}\right)$ & $\bar{\sigma}_{a}[1] \times 10^{5}$ & $\gamma_{1}$ & $\gamma_{2}$ & $T$ \\
\hline (10) Hygiea & S1 & 4484 & 558 & 71.2 & 2.4 & 36.1 & 0.48 \\
(10) Hygiea & S4 & 4348 & 551 & 109.8 & 10.2 & 246.1 & 0.94 \\
(10) Hygiea & S6 & 4410 & 543 & 107.3 & -9.3 & 463.1 & 0.52 \\
(10) Hygiea & S9 & 4422 & 543 & 74.8 & -1.6 & 108.8 & 0.71 \\
(10) Hygiea & All & $(4416 \pm 56)$ & $(548 \pm 7)$ & $(90.8 \pm 20.6)$ & $(0.4 \pm 8.1)$ & $(213.5 \pm 187.8)$ & $(0.66 \pm 0.21)$ \\
(2) Pallas & S1 & 65 & 1 & 14.6 & -2.3 & 11.0 & 0.50 \\
(2) Pallas & S4 & 57 & 1 & 21.9 & -2.4 & 10.3 & 0.61 \\
(2) Pallas & S6 & 69 & 2 & 24.2 & -4.8 & 30.5 & 0.56 \\
(2) Pallas & S9 & 60 & 3 & 22.5 & -3.4 & 12.3 & 0.63 \\
(2) Pallas & All & $(62 \pm 5)$ & $(2 \pm 1)$ & $(20.8 \pm 4.2)$ & $(-3.2 \pm 1.1)$ & $(16.0 \pm 9.7)$ & $(0.57 \pm 0.05)$ \\
(31) Euphrosyne & S1 & 459 & 92 & 61.7 & 3.7 & 36.3 & 0.50 \\
(31) Euphrosyne & S5 & 606 & 138 & 92.5 & 2.9 & 20.4 & 0.54 \\
(31) Euphrosyne & S6 & 474 & 113 & 71.8 & -1.2 & 12.7 & 0.86 \\
(31) Euphrosyne & S7 & 434 & 82 & 65.5 & -10.0 & 12.8 & 0.60 \\
(31) Euphrosyne & All & $(493 \pm 77)$ & $(106 \pm 24)$ & $(72.9 \pm 13.7)$ & $(-1.2 \pm 6.3)$ & $(20.6 \pm 11.1)$ & $(0.63 \pm 0.16)$ \\
\hline
\end{tabular}

Quite surprisingly, considering the results for (2) Pallas, we found a rather high value of mean $\bar{\sigma}_{a}[1]\left(72.9 \times 10^{-5} \mathrm{AU}\right)$, which is higher than the mean value for (4) Vesta in Carruba et al. (2012), but lower than the mean value for (1) Ceres in the same article. This unexpected result shows that there is no simple correlation between high inclination and drift rates by close encounters in proper $a$ and that values of drift rates need to be investigated with a case-by-case approach.

\section{Completeness of the frequency distribution functions}

In the previous section, we obtained several distributions of changes in proper $a$ (frequency distribution functions hereafter) for different massive asteroids and integration schemes. As discussed in Sect. 4, we do observe fluctuations in the numbers of encounters and in the moments of the distributions. A question that may now arise is how far from obtaining a statistically complete sample our results are. Are our frequency distribution functions (or $f d f$ ) of $\Delta a$ values a good approximation of the real probability distribution function ( $p d f$ hereafter), or is a larger sample of close encounters needed to obtain a more complete statistics? And if a larger sample is needed, what could be the minimum number of close encounters needed to obtain a good approximation of the $p d f$ at, for example, a $3 \sigma$ level $^{3}$.

To test how complete our frequency distribution functions are in the interval of $\Delta a$ changes that we are more interested in $\left(0.0006<|\Delta a|<0.006 \mathrm{AU}\right.$, i.e., between $3 \sigma_{\text {noise }}$ values in proper $a$ and ten times this value), we performed KolmogorodSmirnoff probability tests (KS tests hereafter) for each of the observed distributions at confidence levels of $1 \sigma, 2 \sigma$, and $3 \sigma$, with probabilities for the distributions to be compatible of $68.27 \%$, $95.34 \%$, and $99.73 \%$, respectively. Figure 5 shows histograms of frequency of changes in proper $a$ caused by (10) Hygiea (panel A), (2) Pallas (panel B), and (31) Euphrosyne (panel C) in the various integration schemes that we used in this work. None of the obtained distributions was compatible at a $3 \sigma$ level. However, we found that all Hygiea distributions were compatible at a $2 \sigma$ level in this $|\Delta a|$ interval, all Euphrosyne distributions

\footnotetext{
3 By $\sigma$ we mean here the standard deviation of the normal or Gaussian distribution. A $3 \sigma$ level means that there is a $99.73 \%$ probability that the two distributions were compatible.
}

were compatible at $1 \sigma$ level (but only the S1 and S5, and the S6 and the S7 were compatible at a $2 \sigma$ level), and only the S1 and S6 Pallas distributions were compatible at a $1 \sigma$ level. We believe that our results show that we are obtaining a good approximation of the probability distribution function for (10) Hygiea, a decent one for (31) Euphrosyne, and a poor one for (2) Pallas. In our opinion, improving the completeness of our $f d f$ s, possibly to a $3 \sigma$ level, remain a challenge for future works ${ }^{4}$. But, before doing so, we could question what the minimum number of close encounters needed to obtain a more complete statistics could be.

To try answering this question, we turn our attention to the work of Greenberg (1982). In that work, the author computed the change of heliocentric velocity, $\Delta U_{0}$, caused by a close encounter with a massive body as a function of distance at closest approach $r_{\min }$ and relative velocity with respect to the massive asteroid $V$. In Greenberg's model ${ }^{5}$ the sine of the angle $\chi$ at which the asteroid orbit will be deflected due to the close encounter is given by

$\sin \chi=\left[1+\left(\frac{r_{\min } \cdot V^{2}}{G(M+m)}\right)^{2}\right]^{-\frac{1}{2}}$,

where $G$ is the gravitational constant, $M$ the mass of the massive body, and $m$ the (assumed negligible) mass of the incoming asteroid. The change in heliocentric velocity $\Delta U_{0}$ is then given by

$\Delta U_{0}=\left(\frac{M}{M+m}\right) V[\sin 2 \chi \boldsymbol{d}+(\cos 2 \chi-1) \boldsymbol{V}]$,

where $\boldsymbol{d}$ is the unit vector pointing in the direction of $r_{\min }$ and $\boldsymbol{V}$ is the unit vector pointing in the direction of $V$. Figure 6 shows a contour plot of changes in heliocentric velocities as a function of $r_{\min }$ and $V$ for (10) Hygiea (for brevity we do not show analogous plots for (2) Pallas and (31) Euphrosyne). The encounters

\footnotetext{
4 In the case of (2) Pallas, one may question the merit of improving our knowledge of the details of an effect that is quite certainly fairly small. 5 This model is based on a "patching conics" approach, where the encounter is treated as a hyperbolic flyby of the massive body. Such a model would not be able to account for other massive body perturbations, resonant encounters, etc. Since our goal here is to obtain a firstorder approximation of the number of encounters needed to obtain a decent fit of the $p d f$, we believe that our approach is justified.
} 


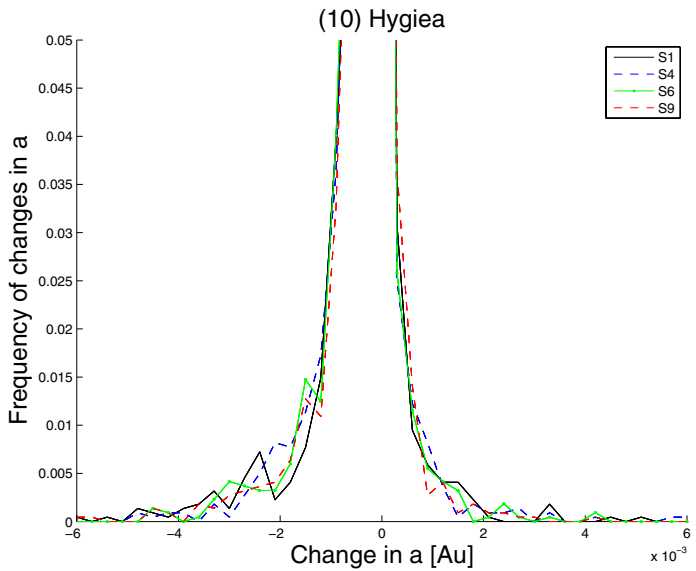

(2) Pallas

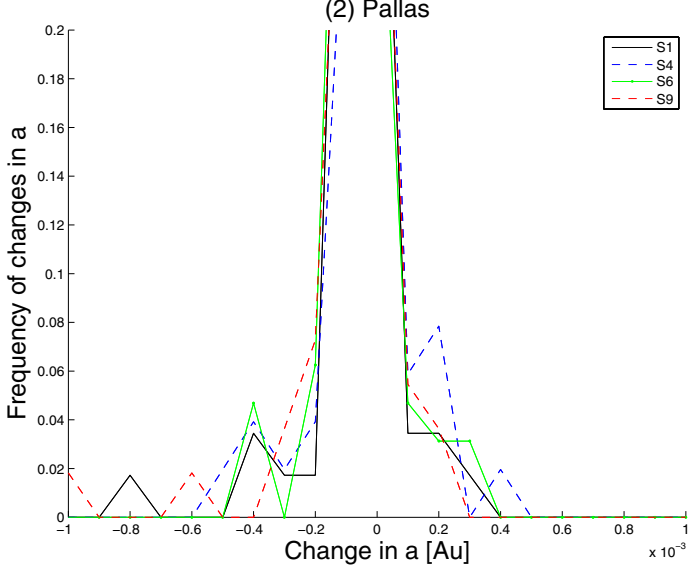

(31) Euphrosyne

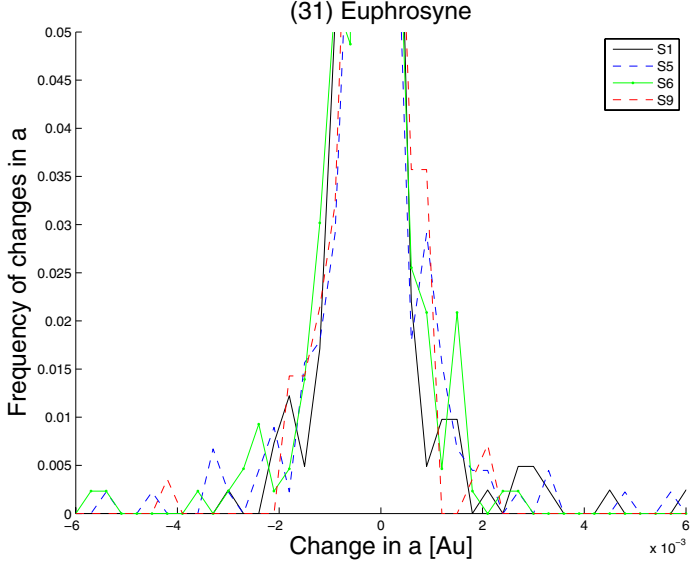

Fig. 5. Histograms of frequency of changes in proper $a$ caused by (10) Hygiea (panel A), (2) Pallas (panel B) and (31) Euphrosyne (panel C) in the integration schemes listed in Table 1.

at low relative velocities and low minimum distances cause the maximum change in heliocentric velocities, and, as discussed in Carruba et al. (2007), the maximum change in heliocentric velocity is of the order of the escape velocity from the massive body (of the order of $200 \mathrm{~m} / \mathrm{s}$ for bodies of the masses and sizes of (2) Pallas, (4) Vesta, and (10) Hygiea).

Since here we are interested in determining the minimum number of close encounters needed to obtain a fairly complete $f d f$ in proper $a$, we used the Gauss equation

$\frac{\Delta a}{a}=\frac{2}{n a\left(1-e^{2}\right)^{1 / 2}}\left[(1+e \cos f) \Delta V_{T}+(e \sin f) \Delta V_{R}\right]$,

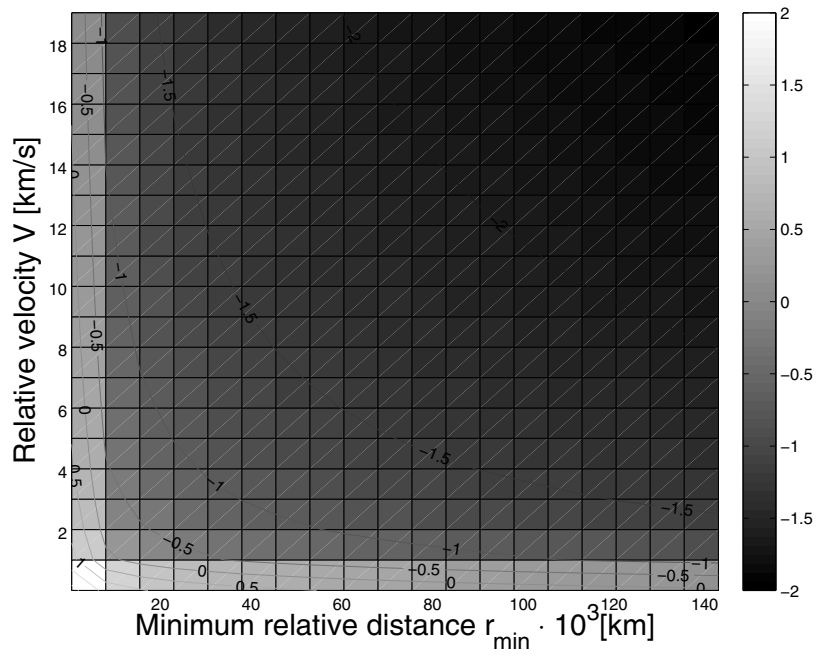

Fig. 6. Contour plot of the $\log _{10}$ of changes in heliocentric velocities $\Delta U_{0}$ as a function of $r_{\min }$ and $V$ for (10) Hygiea according to Eq. (5).

where $\Delta V_{T}, \Delta V_{R}$, (and $\Delta V_{W}$ ) are the tangential, radial, and perpendicular to the orbital plane components of the change in heliocentric velocity $\Delta U_{0}, n$ is the minor body mean-motion, $a$ and $e$ its semi-major axis and eccentricity, and $f$ is the true anomaly at the instant of close encounter. With the simplistic assumptions that the eccentricity of the minor body is zero and that all the change in velocity goes into the tangential component (this last hypothesis may introduce an error of a factor $\sqrt{3}$ ), Eq. (6) reduces to

$\Delta a=\frac{2}{n} \Delta U_{0}$.

We can then use Greenberg's model to compute maximum changes in proper $a$ for different values of $r_{\min }$ and $V$. Figure 7 shows frequency of $\Delta a$ values computed with Eqs. (7) and (5) for different, equally spaced, numbers of close encounters with (10) Hygiea at different and equally spaced values of $r_{\min }$ and $V$ (results are similar for (2) Pallas and (31) Euphrosyne). The values of $r_{\min }$ and $V$ used in Eq. (5) are in the same range of those shown in Fig. 6. Standard KS tests show that the distributions start to be compatible for $\simeq 3000$ equally spaced encounters (we tested distributions for up to 6 million encounters with similar results), which sets a lower limit on the minimum number of close encounters that needs to be tested to obtain a fairly complete $f d f$ and that seems to be in agreement with what we found numerically for the simulations with (10) Hygiea. As a further numerical test, we also computed the standard deviation of changes in $a$ obtained with Greenberg's model for 600, 1500, 3000, and 60000 close encounters. Since this approach only gives absolute values of changes in $a$, the standard deviation of $\Delta a$ does not correspond to drift rates, so we computed the variance of the distributions, rescaled to a mean equal to zero, using this formula

$\sigma_{a}^{2}=\frac{1}{N} \sum_{i=1}^{N}\left((\Delta a-0)^{2}\right)=\frac{1}{N} \sum_{i=1}^{N}\left(|\Delta a|^{2}\right)$,

where $N$ is the number of close encounters. Here we assumed that the mean value of the distributions of changes in $a$ was 0 , which seems to be confirmed by our results of Sect. 4, where the mean values were all less than $10^{-6} \mathrm{AU}$, i.e., less than the numerical error on $\Delta a$. Drift rate values will be equal to the square root 


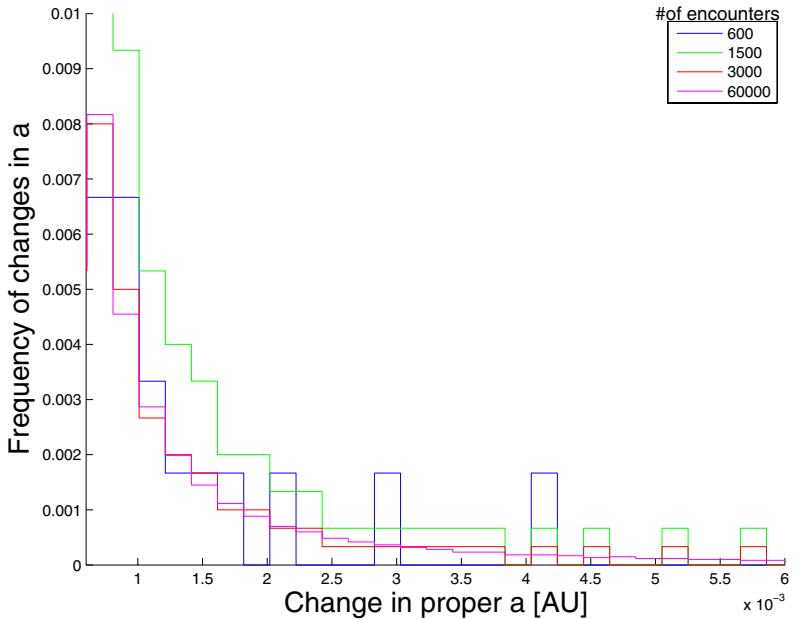

Fig. 7. Histogram of $\Delta a$ changes computed using Greenberg (1982) model for different numbers of close encounters with (10) Hygiea.

of the rescaled variance given by Eq. (8). To more easily compare these results with those of Sect. 4, we also multiplied them by $10^{5}$. We obtained values of drift rates of $230.7,180.1,127.4$, and $102.6 \times 10^{5} \mathrm{AU}$ for the four distributions, with the last two values in the range of what was found in Sect. 4 for the actual simulations with symplectic integrators. KS tests for values of $\Delta a>3 \sigma_{\text {noise }}$ show that the distributions for 3000 and 60000 enncounters are compatible between them, but not with those with 600 and 1500 encounters. Increasing the number of observed close encounters may, of course, improve the completeness of the $f d f$. Obtaining better fits for the $p d f$ of (10) Hygiea and (31) Euphrosyne remains a challenge for future works.

\section{Close encounters with massive asteroids when the Yarkovsky and YORP effects are considered}

In the previous sections, we analyzed the orbital diffusion caused by close encounters with massive asteroids when nongravitational forces such as the Yarkovsky and YORP effects are not considered. Here we further investigate the subject when these effects are taken into account. For this purpose, we use the São Paulo (SP) integrator, which is able to simulate both close encounters with massive asteroids and the Yarkovsky effect. See Carruba et al. (2007) for a treatment of the integrator and of the Yarkovsky effect. Here we just point out that we used the Vokrouhlický model (Vokrouhlický 1999) for the diurnal and seasonal versions of the Yarkovsky effect, so that the drift rate in semi-major axis is given by

$$
\frac{\mathrm{d} a}{\mathrm{~d} t}=k_{1} \cos \epsilon+k_{2} \sin ^{2} \epsilon,
$$

where $k_{1}$ and $k_{2}$ are functions depending on the surface thermal parameters and size, and $\epsilon$ is the spin axis obliquity (see Vokrouhlický 1999, for the exact expressions of the $k_{1}$ and $k_{2}$ functions). In this work, we integrated the same real asteroids with $H<13.5$ in the regions of the Hygiea, Pallas, and Euphrosyne families considered in the previous sections over $30 \mathrm{Myr}$ with two spin obliquities, $\epsilon=0^{\circ}$ and $\epsilon=180^{\circ}$, which maximizes the drift caused by the diurnal version of the Yarkovsky effect. Using Eq. (1), we computed the radius of each object using its absolute magnitude and a geometric albedo equal
Table 3. Number of encounters, $\bar{\sigma}_{a}[1] \times 10^{5}$, and Hurts exponents $T$ for clones of real $H<13.5$ asteroids.

\begin{tabular}{lcccc}
\hline \hline $\begin{array}{l}\text { Asteroid } \\
\text { id. }\end{array}$ & $\begin{array}{c}\text { Spin } \\
\text { orientation }\end{array}$ & $N_{\text {enc }}$ & $\begin{array}{c}\bar{\sigma}_{a}[1] \times 10^{5} \\
{[\mathrm{AU}]}\end{array}$ & $T$ \\
\hline$(10)$ & $\epsilon=0^{\circ}$ & 4880 & $(70.8 \pm 16.3)$ & $\left(0.83_{-0.83}^{+1.16}\right)$ \\
$(10)$ & $\epsilon=180^{\circ}$ & 4719 & $(81.8 \pm 18.8)$ & $\left(0.81_{-0.81}^{+0.84}\right)$ \\
$(10)$ & All & 9599 & $(76.3 \pm 17.5)$ & $(0.82 \pm 0.64)$ \\
\hline$(2)$ & $\epsilon=0^{\circ}$ & 71 & $(7.9 \pm 1.6)$ & $\left(0.63_{-0.63}^{+1.02}\right)$ \\
$(2)$ & $\epsilon=180^{\circ}$ & 66 & $(7.5 \pm 1.5)$ & $\left(0.56_{-0.56}^{+0.74}\right)$ \\
$(2)$ & All & 137 & $(7.7 \pm 1.5)$ & $(0.60 \pm 0.57)$ \\
\hline$(31)$ & $\epsilon=0^{\circ}$ & 508 & $(39.1 \pm 7.3)$ & $\left(0.65_{-0.65}^{+0.95}\right)$ \\
$(31)$ & $\epsilon=180^{\circ}$ & 507 & $(51.5 \pm 9.7)$ & $\left(0.55_{-0.55}^{+0.69}\right)$ \\
$(31)$ & All & 1015 & $(45.3 \pm 8.5)$ & $(0.60 \pm 0.53)$ \\
\hline
\end{tabular}

to 0.1 , a value typical for B- and C-type objects, the most commonly found asteroids in the regions of (2) Pallas, (10) Hygiea, and (31) Euphrosyne. We used Yarkovsky parameters that are also typical for B- and C-type objects: a thermal conductivity $K=0.001 \mathrm{~W} / \mathrm{m} / \mathrm{K}$ (Delbo et al. 2007), a thermal capacity of $680 \mathrm{~J} / \mathrm{kg} / \mathrm{K}$, a surface density of $1500 \mathrm{~kg} / \mathrm{m}^{3}$, a bulk density of $1500 \mathrm{~kg} / \mathrm{m}^{3}$, a Bond albedo of 0.1 , and a thermal emissivity of 0.95 (see also Carruba et al. 2003, for a more in-depth discussion of these parameters).

Following the approach of Delisle \& Laskar (2012), we assumed that the obliquity remains constant during a YORP cycle, with maximal values of $\epsilon=0^{\circ}$ or $\epsilon=180^{\circ}$, which yields a maximum strength for the diurnal version of the Yarkovsky effect and a minimal strength for the seasonal one. We also assumed that re-orientations act almost instantaneously at the end of each YORP cycle, with an assumed timescale of $30 \mathrm{Myr}$ for $\mathrm{km}$-sized objects (Delisle \& Laskar 2012). We integrated our real asteroids using our S1 and S0 integration schemes (the latter was used to estimate the values of $\sigma_{\text {noise }}$, as discussed in the previous section), and we used the values of the standard deviations on the moments obtained in the previous section to estimate the possible errors associated with the particular integration scheme used in this work.

As in Sect. 4, we used the three values of $\bar{\sigma}_{a}[1](t)$ computed at each $10 \mathrm{Myr}$ to determine the $T$ value of the Hurst exponent. However, with respect to previous works, we also account for the uncertainty associated with changes in $\bar{\sigma}_{a}[1](t)$ between integrations with different schemes in the following manner: assuming that errors in $\bar{\sigma}_{a}[1](t)$ are of the order of the standard deviations found in Sect. 4 for (10) Hygiea, (2) Pallas, (31) Euphrosyne, i.e. $22.7 \%, 20.0 \%$, and $18.8 \%$ of the mean value, respectively, we can estimate the value of $T$ and its error using standard techniques of linear regression (see Press et al. 2001, Eqs. (15.2.4), (15.2.6), and (15.2.9)).

Table 3 shows values of number of encounters $N_{\text {enc }}$, of $\bar{\sigma}_{a}[1] \times 10^{5} \mathrm{AU}$, and Hurts exponents $T$ for (10) Hygiea, (2) Pallas, and (31) Euphrosyne for (i) the simulation with clones of real asteroids with initial zero obliquity; (ii) the simulation with clones of real asteroids with $\epsilon=180^{\circ}$; and iii) for all asteroid clones. For the sake of brevity, we do not report the other moments $\gamma_{1}$ and $\gamma_{2}$ listed in Table 2. Errors on $\bar{\sigma}_{a}[1] \times 10^{5}$ are assumed of the order of what was found in Sect. 4. Values of $\bar{\sigma}_{a}[1] \times 10^{5}$ for the simulations with the São Paulo integrator are in the same range (to within the errors) of what was found for the conservative case in Sect. 4 for (10) Hygiea, but somewhat smaller for (2) Pallas and (31) Euphrosyne. This fact may be caused by the limited number of close encounters that occurred during the simulations for these asteroids, which was 
considerably lower than the completeness level of $\simeq 3000$ encounters found in Sect. 5 for the $f d f$ to converge. Also, while the $T$ values that best fitted our data are still compatible with scattering with massive asteroids being a persistent and correlated process, we unfortunately confirm that, as found in Carruba et al. (2012), the uncertainties are so large that no final conclusions can be positively achieved.

\section{Conclusions}

We studied the problem of orbital diffusion in semi-major axis of minor bodies for the case of encounters with (10) Hygiea, (2) Pallas, and (31) Euphrosyne. Our main results can be summarized as follows:

- We obtained the proper frequencies of precession of the argument of pericenter $g$ and of longitude of the node $s$ for the three massive asteroids when other massive asteroids (up to 38 ) are considered along with the eight planets. The values fluctuated beyond a $3 \sigma$ confidence level for various integration schemes, such as the S9 and S6. Lower fluctuations were also observed when different numbers of massive asteroids were considered.

- We investigated the dynamical mobility caused by close encounters with massive asteroids for real asteroids in the orbital regions of (10) Hygiea, (2) Pallas, and (31) Euphrosyne with absolute magnitude $H<13.5$. As in Carruba et al. (2012), we found that not only different asteroids experienced close encounters with the three massive bodies when different integration schemes were considered, but also that the whole statistics of close encounters fluctuated. Variances of the change in proper $a$ massive asteroids varied up to $23 \%$ in the four integration schemes that we used. We confirm the hypothesis of Bottke et al. (1994) that encounters with highly inclined objects as (2) Pallas are indeed a minor effect because of rather high relative speed and distances at encounters between perturber and perturbee. Unexpectedly, we found a very large mean value of drift rate in proper $a$ caused by close encounters with (10) Hygiea, $\bar{\sigma}_{a}[1] \times 10^{5}=$ $(90.8 \pm 20.6) \mathrm{AU}$, and (31) Euphrosyne, $\bar{\sigma}_{a}[1] \times 10^{5}=$ $(72.9 \pm 13.7)$ AU. These values are higher than the drift rate caused by (4) Vesta for its orbital region $\left(\bar{\sigma}_{a}[1] \times 10^{5}=\right.$ $(29.5 \pm 10.7) \mathrm{AU})$, as found in Carruba et al. (2012), and may have repercussion on the orbital evolution of members of their respective families.

- We performed KS tests on the completeness of the $f d f$ obtained for the three massive asteroids. The frequency distributions obtained with the different integration schemes are compatible among themselves to a $2 \sigma$ level for (10) Hygiea, to a $1 \sigma$ level for (31) Euphrosyne, and to lower levels for (2) Pallas. Considerations based on the model of Greenberg (1982) suggest that the minimum number of close encounters needed to obtain a good fit of the $p d f$ are of the order of 3000, with expected uncertainties on the measured drift rates of the order of $10 \%$ for this number of encounters. Obtaining better fits of the $p d f$ for (10) Hygiea and (31) Euphrosyne, possibly to a $3 \sigma$ level, remains a challenge for future works.

- We studied the effect of close encounters with massive asteroids when the Yarkovsky and YORP effects were also considered and computed the value of the Hurst exponent $T$ of the diffusion as a function of time, including the effect of the uncertainty caused by differences in the integration schemes as found in this work. Drift rate values and Hurst exponents for the Hygiea region are in the same range, to within the errors, as those found in the conservative integrations (for the Pallas and Euphrosyne areas, they are somewhat smaller, but this may be due to the limited number of close encounters that occurred during the simulations for asteroids in these regions), and Hurst exponents indicate scattering with massive asteroids being a persistent and correlated process $(0.5<T<1.0$, but values are affected by large uncertainties). This suggests that in order to obtain estimates of these parameters on the timescales used in this work (30 Myr), including the Yarkovsky and YORP effects may not be necessary.

In this work we investigated for the first time the long-term effect that close encounters with massive asteroids such (10) Hygiea, (2) Pallas, and (31) Euphrosyne may have had on the orbital evolution of asteroids in their orbital proximity. In particular, the unexpected high rate of drift in proper $a$ caused by encounters with (10) Hygiea and (31) Euphrosyne, unknown in previous works in the literature, suggests that this diffusion mechanism may have played a significant role in the evolution of their respective families that is yet to be investigated. Also, the relative large value of drift rate found for (31) Euphrosyne shows that there is no simple correlation between the high inclination of the perturber and the drift rates caused by close encounters. According to our results, values of drift rates need therefore to be investigated with a case-by-case approach.

Acknowledgements. We are grateful to the anonymous referee of this paper for comments and suggestions that significantly improved the quality of our work. We also thank G. Valsecchi for discussions and hints that opened interesting new lines of research. This work was supported by the Brazilian National Research Council (CNPq), project 305453/2011-4, by the Foundation for Supporting Scientific Research in the São Paulo state (FAPESP), grant 11/19863-3, and by the Coordenation for Improvement of Higher Education Personnel (CAPES), grant 23038.007093/2012-13.

\section{References}

Baer, J., Chesley, S. R., \& Matson, R. D. 2011, AJ, 141, 143

Bottke, W. F., Nolan, M. C., Greenberg, R., \& Kolvoord, R. A. 1994, Icarus, 107, 255

Bowell, E., Hapke, B., Domingue, D., et al. 1989, in Asteroids II (Tucson: Univ. of Arizona), 524

Carruba, V. 2010, MNRAS, 408, 580

Carruba, V., \& Machuca, J. F. 2011, MNRAS, 420, 1779

Carruba, V., \& Michtchenko, T. A. 2007, A\&A, 475, 1145

Carruba, V., \& Morbidelli, A. 2011, MNRAS, 412, 2040

Carruba, V., Burns, J. A., Bottke, W., \& Nesvorný, D. 2003, Icarus, 162, 308

Carruba, V., Roig, F., Michtchenko, T. A., Ferraz-Mello, S., \& Nesvorný, D. 2007, A\&A, 465, 315

Carruba, V., Huaman, M., Douwens, S., \& Domingos, R. C. 2012, A\&A, 543, A105

Carry, B. 2012, Planet. Space Sci., accepted [arXiv: 1203 .4336]

Delisle, J.-B., \& Laskar, J. 2011, A\&A, 540, A18

Knežević, Z., \& Milani, A. 2003, A\&A, 403, 1165

Greenberg, R. 1982. AJ, 87, 184

Laskar, J. 1988, A\&A, 198, 341

Laskar, J. 1990, Icarus, 88, 266

Laskar, J. 1993, Phys. Rev. Lett. 70, 2975

Levison, H. F., \& Duncan, M. J. 2000, AJ, 120, 2117

Milani, A., \& Knežević, Z. 1994, Icarus, 107, 219

Machuca, J. F., \& Carruba, V. 2011, MNRAS, 420, 1779

Press, V. H., Teukolsky, S. A., Vetterlink, W. T., \& Flannery, B. P. 2001, Numerical Recipes in Fortran 77 (Cambridge: Cambridge Univ. Press)

Russell, C. T., Raymond, C. A., Coradini, A., et al. 2012, Science, 336, 684

Šidlichovský, M., \& Nesvorný, D. 1997, Celest. Mech. Dyn. Astron., 65, 137 


\section{Appendix A: Asteroid astrometric masses}

We report in Table A.1 the identification of each asteroid used in this work, its mass and mass uncertainty as in Carry (2012), and the percentile errors associated with each asteroid mass (see Carry 2012, for a discussion of the methods used to determine the asteroid masses, and their uncertainties).

Table A.1. Asteroid astrometric masses with their uncertainties.

\begin{tabular}{|c|c|c|c|}
\hline Asteroid & Mass (in $10^{15} \mathrm{~kg}$ ) & Mass uncertainty (in $10^{15} \mathrm{~kg}$ ) & Percentile error \\
\hline 1 Ceres & 944000 & 6000. & 0.006 \\
\hline 4 Vesta & 259000. & 0. & 0.000 \\
\hline 2 Pallas & 204000. & 4000. & 0.020 \\
\hline 10 Hygiea & 86300. & 5200. & 0.060 \\
\hline 511 Davida & 33800. & 10200. & 0.302 \\
\hline 704 Interamnia & 32800. & 4500. & 0.137 \\
\hline 15 Eunomia & 31400. & 1800. & 0.057 \\
\hline 3 Juno & 27300. & 2900. & 0.106 \\
\hline 16 Psyche & 27200. & 7500. & 0.276 \\
\hline 536 Merapi & 26100. & 4700. & 0.180 \\
\hline 52 Europa & 23800. & 5800. & 0.244 \\
\hline 165 Loreley & 19100. & 1900. & 0.099 \\
\hline 88 Thisbe & 15300. & 3100. & 0.203 \\
\hline 87 Sylvia & 14800. & 0. & 0.000 \\
\hline 420 Bertholda & 14800. & 900. & 0.061 \\
\hline 6 Hebe & 13900. & 1000. & 0.072 \\
\hline 65 Cybele & 13600. & 3100. & 0.228 \\
\hline 212 Medea & 13200. & 1000. & 0.076 \\
\hline 7 Iris & 12900. & 2100. & 0.163 \\
\hline 29 Amphitrite & 12900. & 2000. & 0.155 \\
\hline 690 Wratislavia & 12800. & 300. & 0.023 \\
\hline 31 Euphrosyne & 12700. & 6500. & 0.512 \\
\hline 57 Mnemosyne & 12600. & 2400. & 0.190 \\
\hline 147 Protogeneia & 12300. & 500. & 0.041 \\
\hline 675 Ludmilla & 12000. & 2400 & 0.200 \\
\hline 409 Aspasia & 11800. & 2300 . & 0.195 \\
\hline 532 Herculina & 11500. & 2800. & 0.243 \\
\hline 107 Camilla & 11200. & 300. & 0.027 \\
\hline 451 Patientia & 10900. & 5300. & 0.486 \\
\hline 200 Dynamene & 10700. & 1600. & 0.150 \\
\hline 444 Gyptis & 10600. & 2800. & 0.264 \\
\hline 324 Bamberga & 10300. & 1000. & 0.097 \\
\hline 602 Marianna & 10200. & 500. & 0.049 \\
\hline 895 Helio & 9870. & 6050. & 0.613 \\
\hline 154 Bertha & 9190. & 5200. & 0.566 \\
\hline 381 Myrrha & 9180. & 800. & 0.087 \\
\hline 8 Flora & 9170. & 1750. & 0.191 \\
\hline 13 Egeria & 8820. & 4250. & 0.482 \\
\hline 19 Fortuna & 8600. & 1460. & 0.170 \\
\hline
\end{tabular}

